\title{
5. Die Abteilung für Naturheilkunde in der Klinik Blan- kenstein, Hattingen, mit dem Lehrbereich für Natur- heilkunde und Gesundheitsprävention an der Ruhr- Universität Bochum
}

\section{ANDRÉ-MICHAEL BEER}

In der Abteilung Naturheilkunde der Klinik Blankenstein werden vornehmlich Chronisch- und Langzeiterkrankte, insbesondere mit psychosomatisch-psychologischer Komponente in der Krankheitsverarbeitung, mit klassischen Naturheilverfahren stationär behandelt. Die naturheilkundliche Komplexbehandlung mit den klassischen Naturheilverfahren, seriell und in hoher Dichte angewendet, führt dabei zum Erfolg. Seit Eröffnung der Abteilung im Jahre 1997 wird immer wieder empirisch erfahren, wie erfolgreich die kombinierte stationäre Behandlung von Naturheilverfahren und konventioneller Medizin ist.

Neben den Abteilungen der Inneren Medizin, Chirurgie, Orthopädie und Anästhesie (Schmerztherapie) gibt es in Blankenstein die Abteilung für Naturheilkunde mit 6o Krankenhausbetten. Die Klinik Blankenstein gehört zum Klinikverbund Katholische Klinikum gGmbH.

Haus- oder Fachärzte können, sofern die medizinische Notwendigkeit für einen stationären Aufenthalt besteht, ihre Patienten in die Abteilung Naturheilkunde einweisen.

Die Abteilung wird von Patienten aus der näheren und ferneren Umgebung seit Jahren gut angenommen und weist eine volle Auslastung auf. Abhängig von der DRG dauert der Aufenthalt zwischen 13 und 18 Tage.

Entsprechend den Wirkweisen von Naturheilverfahren als Reiz-Reaktionstherapien ist dieser Zeitraum notwendig, um Erfolge $z u$ erzielen. Oberstes Ziel 
von Naturheilverfahren ist es, die Selbstheilungskräfte des Körpers anzuregen, was in der Regel ein Ausschöpfen der oberen Verweildauer erfordert.

Die Abteilung für Naturheilkunde sieht neben ihrer täglichen Arbeit in der Patientenversorgung die Lehre und Forschung als wesentliche Schwerpunkte.

Für die Patientenversorgung steht ein interdisziplinäres Team zur Verfügung, das den hohen Anforderungen, die an die Versorgung der meist chronisch Erkrankten gestellt werden, gerecht wird. Die Patienten werden unter Einbeziehung der medizinischen Gesamtkompetenz, die im Klinikverbund Katholische Klinikum Bochum gGmbH zur Verfügung steht, behandelt. Die klinische Zusammenarbeit garantiert die Möglichkeit der Hinzuziehung verschiedenster Fachspezialisten aus der Universitätsklinik. Es handelt sich um die Bündelung von Kompetenz, die die naturheilkundliche Versorgung mit einschließt.

Somit stehen den Ärzten der Abteilung für Naturheilkunde nahezu alle medizinischen Fachrichtungen konsiliarisch zur Seite.

In der Abteilung Naturheilkunde arbeiten insgesamt 5 Ärzte und Ärztinnen mit unterschiedlichen Facharztbezeichnungen, (u.a. Innere Medizin, Allgemeinmedizin, Pädiatrie, Frauenheilkunde). Es bestehen Zusatzqualifikationen in Physikalischer Therapie, Rehabilitationsmedizin, Akupunktur, Balneologie und physikalischer Therapie, Psychotherapie und Psychosomatik, Homöopathie, Akupunktur, Onkologie, Sportmedizin, Ernährungsmedizin, manueller Medizin, Chirotherapie und Neuraltherapie. Der Bereich der Ordnungstherapie wird durch diplomierte Psychologinnen und Psychologen vertreten. Das pflegerische Personal führt neben den üblichen pflegerischen Tätigkeiten auch therapeutische Anwendungen (Meerrettichwickel, Heusackauflagen, atemstimulierende Einreibungen etc.) durch. Das therapeutische Team wird vervollständigt durch Oecotrophologinnen und ein physiotherapeutisches Team. In den täglichen Teamsitzungen wird das individuelle Behandlungskonzept vom aufnehmenden Arzt vorgestellt und entsprechend des Beschwerdeverlaufes des Patienten modifiziert.

\subsection{Indikationen}

Schwerpunktindikationen für die stationäre Behandlung sind:

- Rheumatische Erkrankungen (u.a. Rückenschmerzen, Fibromyalgie, rheumatische Arthritis)

- Stoffwechselerkrankungen (u.a. Fettstoffwechselbeschwerden, metabolisches Syndrom)

- Funktionelle Magen/Darmleiden (u.a. Reizdarm, Reizmagen, Magenschleimhautentzündung)

- Chronische Infekte (u.a. Nasennebenhöhlenbeschwerden, chron. Blasenentzündungen)

- Allergische Erkrankungen (u.a. Heuschnupfen, allergisches Asthma) 
- Schmerzzustände

- Krebserkrankungen

- Frauenleiden (u.a. Wechseljahresbeschwerden, Verwachsungsschmerzen)

- Hauterkrankungen (u.a. Neurodermitis, Urticaria)

\subsection{Diagnostische Verfahren}

Die Diagnostik stützt sich auf eine ausführliche Anamnese, eine klinische Untersuchung mit besonderem Augenmerk auf den Magen-Darm-Trakt, die Fokussuche und die ganzheitliche Zahnmedizin. Im Einzelfall werden bei der Therapieauswahl Kriterien der europäischen Konstitutionsmedizin mit einbezogen.

Unter Berücksichtigung der bereits erfolgten Vordiagnostik werden folgende Verfahren bei Bedarf zur Aufnahme- und Verlaufsdiagnostik durchgeführt:

- Naturheilkundliche Labordiagnostik

- Klinisch-chemisches Labor

- Hämatologie

- klinische Chemie, Enzymdiagnostik

- Hormonanalysen

- rheumatologische und immunologische Diagnostik

- Mikrobiologische Diagnostik

- 24-Stunden-Blutdrucküberwachung und 24-Stunden-EKC

- Sonographie (Abdomen, intrakavitäre Sonographie, Gelenke, Gefäßdopplersonographie)

- Ruhe-EKC

- Ergometrie

- Lungenfunktionsprüfung (Spiroergometrie)

- Röntgendiagnostik, MRT, CT, Szintigraphie

- Osteodensitometrie

- Endoskopische Untersuchungen: Rekto-, Prokto-, Kolo-, Gastro-, Zystoskopie

\subsection{Therapeutische Verfahren}

Die Patienten werden mit den in weiten Bereichen wissenschaftlich fundierten oder empirisch plausiblen klassischen Naturheilverfahren (Wasser-, Pflanzen-, Wärme- und Kälteanwendungen, Ernährungstherapie [u.a. Heilfasten], Ordnungstherapie), den erweiterten Naturheilverfahren (u.a. Blutegeltherapie, Schröpfen, Neuraltherapie, und komplementärmedizinischen Verfahren [u.a. Akupunktur] behandelt). 
Der Einsatz der Phytotherapie stellt einen Behandlungsschwerpunkt dar (Phytopharmaka, Medizinaltees, Infusionen mit Phytotherapeutika etc.). Die Ordnungstherapie, die den Patienten dabei unterstützen soll, wieder zu seiner ,inneren Mitte" $z u$ finden, macht einen weiteren wesentlichen Therapieschwerpunkt aus. Sie führt zu besonders günstigen Erfolgen in der Behandlung psychosomatischpsychologischer Krankheitsbilder, die in hohem Maße mit chronifizierten Erkrankungen einhergehen.

In der Gruppenarbeit erfahren die Patienten, wie man aktiv seinen Gesundheitszustand erhalten bzw. verbessern kann. So wird beispielsweise in der Pflanzenheilkundegruppe gelehrt, wie Tees selbst zubereitet werden. In der Ordnungstherapie-, Resilienz- und Schmerztherapiegruppe werden Hilfsmittel an die Hand gegeben, wie der Patient aktiv etwas zu seiner Gesundung beitragen kann und damit seine Krankheitssymptome reduzieren kann. In der Schmerzgruppe erlernen die Patienten Wege, mit ihrem Schmerz besser umgehen zu können (s. Abb. 1).

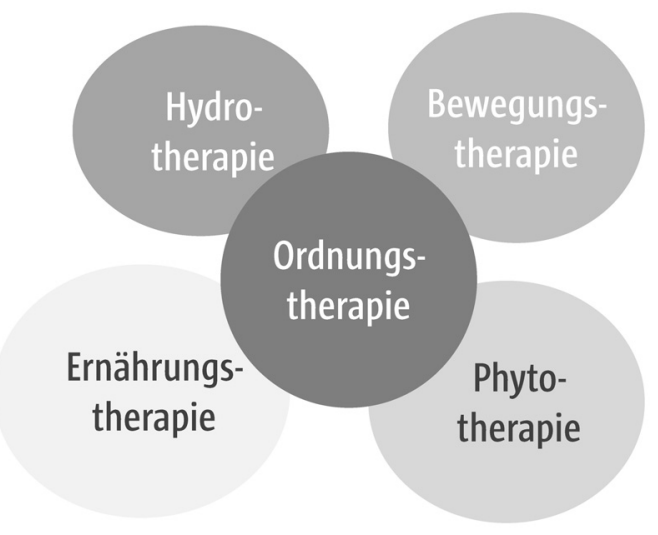

Abb. 1 Klassische Naturheilverfahren

\subsection{Kostenübernahme}

Die Abteilung Naturheilkunde der Klinik Blankenstein ist mit 6o Betten in den Krankenhausplan des Bundeslandes Nordrhein-Westfalens aufgenommen worden.

Versicherte bei Krankenkassen haben Anspruch auf vollstationäre Behandlung nach $\mathbb{3} 39$ SGB V, wenn nach Prüfung durch das Krankenhaus die Aufnahme erforderlich ist, weil das Behandlungsziel nicht durch teilstationäre, vor- und 
nachstationäre oder ambulante Behandlung erreicht werden kann. Privat versicherte Patienten beantragen vor Antritt der medizinisch notwendigen stationären Heilbehandlung bei ihrer Krankenversicherung eine schriftliche Kostenzusage. Die Klinik hat die Beihilfefähigkeit nach $\$ 7$ Abs. 4 BhV und $\$ 6$ Abs. 1 Nr. 6 BhV. Beihilfeberechtigte Patienten müssen die Leistungszusage bei ihrer zuständigen Beihilfestelle vor Antritt des stationären Aufenthaltes einholen.

\subsection{Patientennachfrage}

Die hohe Nachfrage nach der stationären Aufnahme führt regelmäßig zu Wartezeiten. 80\% der Patienten kommen zur stationären Aufnahme auf Anraten des Arztes, der dann die Einweisung ausstellt. Etwa 20\% der Aufnahmen erfolgen auf Eigeninitiative der Patienten.

\subsection{Lehre und Forschung}

Die Lehrabteilung Naturheilkunde ist im Bereich der Forschung und Lehre eng mit der Ruhr-Universität Bochum verbunden.

Der Lehrbereich Naturheilkunde und Gesundheitsprävention der Ruhr-Universität Bochum führt die Hauptvorlesungen zum Querschnittsfach 12 (Rehabilitation, Physikalische Medizin und Naturheilverfahren) sowohl im Regel- als auch im Modellstudiengang durch. Daneben werden regelmäßig Wahlfächer angeboten (u.a. Akupunktur, Phytotherapie). Weiterhin besteht für die Studierenden die Möglichkeit Promotionsarbeiten durchzuführen.

Die Kurse zur Zusatz-Weiterbildung „Naturheilverfahren“ werden seit April 1997 gemeinsam mit der Ärztekammer, der KV Westfalen-Lippe und dem Bereich Naturheilkunde an der Ruhr-Universität Bochum regelmäßig zweimal im Jahr für jeweils 1o Tage durchgeführt.

In der Klinik Blankenstein wurde 2012, in Anlehnung an bereits bestehende österreichische und schweizerische Ausbildungsgänge, eine zertifizierte praxisorientierte Ausbildung für Ärzte zur Phytotherapie etabliert. Die Zertifizierung wird durch den Zentralverband der Ärzte für Naturheilverfahren und Regulationsmedizin e.V. (ZAEN) vorgenommen.

Seit mehr als 15 Jahren wird experimentelle Forschung über pflanzliche Vielstoffgemische unter Anwendung unterschiedlicher pharmakologischer Methoden in einem eigenen Labor durchgeführt. Untersucht werden Wirkungen und Wirkmechanismen von Vielstoffgemischen auf molekularer Ebene. Das therapeutische Potenzial sowohl bekannter als auch bisher noch unerforschter Pflanzen- und Torfinhaltsstoffe wird im Rahmen dieser experimentellen Forschung untersucht. Außerdem werden regelmäßig klinische Studien durchgeführt und die Ergebnisse in entsprechenden Fachzeitschriften publiziert. 
Auch Projekte zur Versorgungsforschung wurden und werden umgesetzt.

Aus der Begleitforschung durch die Universitäten Witten-Herdecke und Bochum liegt eine Vielzahl von aussagefähigen Ergebnissen vor. Die Ergebnisse aus der 3bzw. 6-monatigen Nachbefragung unserer behandelten Patienten zeigen u.a., dass ca. $80 \%$ der von uns behandelten Patienten auch noch nach $1 / 2$ Jahr eine deutliche Beschwerdebesserung sowohl im körperlichen, als auch im seelischen Bereich angeben.

Weiterhin konnte gezeigt werden, dass ein spezifisches chronisch krankes Patientenkollektiv erfolgreich versorgt wird, für das es im Gesundheitswesen derzeit sonst keine adäquate Alternative gibt. Der interdisziplinäre Vergleich der Behandlungsergebnisse zwischen naturheilkundlicher Komplextherapie und universitären orthopädischen und rheumatologischen Fachabteilungen machte deutlich, dass die stationäre naturheilkundliche Komplexbehandlung eine kostengünstige Ergänzung der konventionellen orthopädischen und rheumatologischen Behandlungsoption darstellt. Für bestimmte multimorbide Patientengruppen besteht im Hinblick auf klinische und finanzielle Aspekte ein deutlicher Zusatznutzen der Naturheilkunde.

Insgesamt, so die Ergebnisse, generieren die Naturheilverfahren nachweislich mehr Gesundheitsbewusstsein beim Patienten. Dadurch erfolgt ein Abbau von Risikofaktoren mit langfristigen positiven Folgen und somit geringeren Kosten.

\subsection{Ambulanz für Naturheilkunde}

In der Ambulanz für Naturheilkunde der Klinik Blankenstein kommen klassische Naturheilverfahren und Akupunkturverfahren zur Anwendung. 